\title{
Flavour Violating Axions
}

\author{
Luca Di Luzio ${ }^{1, *}$ \\ ${ }^{1}$ Deutsches Elektronen-Synchrotron DESY, Notkestraße 85, D-22607 Hamburg, Germany
}

\begin{abstract}
I review the physics case for flavour violating axions. In particular, I argue that relaxing the assumption of the universality of the Peccei-Quinn current opens up new pathways, including: the relaxation of the Supernova bound on the axion mass, a possible connection with the Standard Model flavour puzzle and the experimental opportunity of discovering the axion via flavoured axion searches.
\end{abstract}

\section{Introduction}

Axion physics has entered a new, experimentally driven phase. In the recent years we have witnessed the emergence of several new experimental proposals, which (in different stages of development) promise to open for exploration regions of parameter space considered unreachable until few years ago (for recent overviews see Refs. [1, 2]). It is hence timely, on the theory side, to reassess axion properties beyond standard scenarios. In this contribution, I first review some basics aspects of axion physics. The emphasis is put on the fact that axion couplings are inherently UV dependent, so that a wide experimental program should keep this into account, regardless of theoretical prejudice. In particular, I will focus on a commonly adopted, although not strongly motivated, assumption which consists in the universality of the Peccei-Quinn (PQ) current. I will argue, both with old examples and more recent ones, that relaxing the latter assumption is motivated by various phenomenological and theoretical arguments. Most importantly, it offers a unique experimental handle which consists in flavour-violating (FV) axion transitions. The latter turn out to be complementary to standard axion searches and potentially competitive with astrophysical bounds.

\section{Axion couplings: model-independent vs. model-dependent}

The essence of the axion solution of the strong CP problem is a new spin-0 field $a(x)$, the axion, endowed with a pseudo-shift symmetry $a \rightarrow a+\alpha f_{a}$ that is broken only by the operator

$$
\frac{a}{f_{a}} \frac{\alpha_{s}}{8 \pi} G \tilde{G}
$$

While the QCD $\theta$ term can be rotated away via an appropriate choice of $\alpha$, a Vafa-Witten theorem [3] ensures that in a vector-like theory such as QCD, $E(\langle a\rangle=0) \leq$
$E(\langle a\rangle \neq 0)$, thus solving the strong CP problem. The origin of the $a G \tilde{G}$ operator in Eq. (1) can be traced back in the existence of a QCD-anomalous, global U(1) $)_{\mathrm{PQ}}$ symmetry, whose spontaneous breaking delivers the axion as a pseudo-Nambu-Goldstone boson.

The $a G \tilde{G}$ operator represents the smoking-gun signature of the PQ mechanism and determines the so-called model-independent properties of the axion field, which are given solely in terms of the axion decay constant $f_{a}$. These are the axion mass $m_{a}=5.691(51) \mu \mathrm{eV}\left(10^{12} \mathrm{GeV} / f_{a}\right)$ [4], and the axion couplings to photons, nucleons and electrons (here we focus on the most relevant ones for astrophysical limits), arising from the diagrams in Fig. (1).
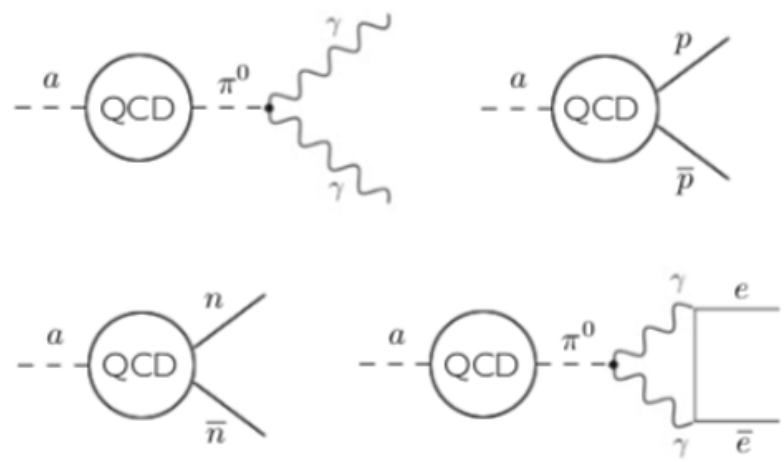

Figure 1. Model-independent axion couplings. The blob stands for non-perturbative QCD dynamics.

Given the effective axion Lagrangian

$$
\mathcal{L}_{a} \supset \frac{\alpha}{8 \pi} \frac{C_{\gamma}}{f_{a}} a F \tilde{F}+\frac{C_{f}}{2 f_{a}} \partial_{\mu} a \bar{f} \gamma^{\mu} \gamma_{5} f, \quad(f=p, n, e),
$$

the numerical values of the coefficients $C_{\gamma, p, n, e}$ can be determined via chiral Lagrangian techniques, as well as inputs from Lattice QCD, and they are found to be [5-7]

$$
\begin{aligned}
& C_{\gamma}=-1.92(4), \quad C_{p}=-0.47(3), \quad C_{n}=-0.02(3), \\
& C_{e}=-7.8(2) \times 10^{-6} \log \left(\frac{f_{a}}{m_{e}}\right) .
\end{aligned}
$$

*e-mail: luca.diluzio@desy.de 
However, being the description of the effective operator in Eq. (1) valid only until energies of the order of $f_{a}$, the theory must be UV completed. Remarkably, the UV completion of the axion effective Lagrangian can drastically affect the low-energy properties of the axion, and hence the way to experimentally probe it.

There are basically two main ways in which this can happen, as depicted schematically in the diagrams of Fig. (2).
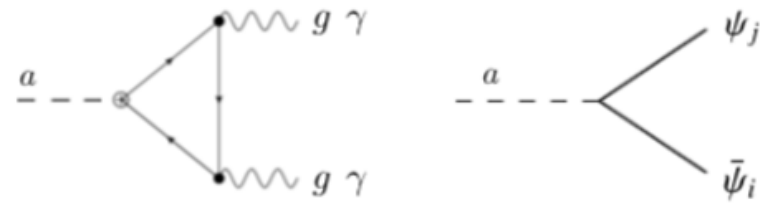

Figure 2. Model-dependent axion couplings to photons and SM quarks and leptons.

In the left diagram of Fig. (2), the PQ-charged colored fermions responsible for generating the $a G \tilde{G}$ operator can also lead to a direct QED-anomalous contribution to $a F \tilde{F}$, if the new fermions they are charged under $\mathrm{U}(1)_{\mathrm{EM}}$. Then the axion coupling to photons gets modified into $C_{\gamma}=E / N-1.92(4)$, where $E / N$ is a group theory factor which depends on the quantum numbers of the fermions running in the loop (see e.g. Refs. [8, 9] for phenomenologically motivated ranges of $E / N$ ).

The other possibility, depicted in the right diagram of Fig. (2), is that the axion interacts directly with the Standard Model (SM) fermions, which are charged under the $\mathrm{U}(1)_{\mathrm{PQ}}$. In this case, the axion effective interaction can be written as (keeping for the sake of illustration only SM quarks)

$$
\begin{aligned}
\frac{\partial_{\mu} a}{v_{\mathrm{PQ}}} J_{\mathrm{PQ}}^{\mu} & =\frac{\partial_{\mu} a}{v_{\mathrm{PQ}}} \\
& \times\left[-\bar{Q}_{L} \mathcal{X}_{Q_{L}} \gamma_{\mu} Q_{L}-\bar{u}_{R} \mathcal{X}_{u_{R}} \gamma_{\mu} u_{R}-\bar{d}_{R} \mathcal{X}_{d_{L}} \gamma_{\mu} d_{R}\right],
\end{aligned}
$$

where $J_{\mathrm{PQ}}^{\mu}$ is the conserved (up to anomalies) PQ current, depending on the $\mathrm{U}(1)_{\mathrm{PQ}}$ charges. The latter are denoted by $\mathcal{X}_{Q_{L}, u_{R}, d_{R}}$, which are diagonal (in general, nonuniversal) matrices. After going to the mass basis: $u_{L} \rightarrow$ $V_{u_{L}} u_{L}$, etc., and using the relation $f_{a}=v_{\mathrm{PQ}} /(2 N)$ between the axion decay constant and the PQ-breaking order parameter, we can recast Eq. (4) as

$$
\frac{\partial_{\mu} a}{2 f_{a}} \bar{\psi}_{i}\left(C_{\psi_{i} \psi_{j}}^{V}+C_{\psi_{i} \psi_{j}}^{A} \gamma_{5}\right) \gamma^{\mu} \psi_{j},
$$

where mass eigenstates are denoted as $\psi_{i}=\left\{u_{i}, d_{i}\right\}$ and we have introduced the vector ${ }^{1}$ and axial couplings

$$
\begin{aligned}
& C_{u_{i} u_{j}}^{V, A}=\frac{1}{2 N}\left(V_{u_{L}}^{\dagger} \chi_{Q_{L}} V_{u_{L}} \pm V_{u_{R}}^{\dagger} X_{u_{R}} V_{u_{R}}\right)_{i j}, \\
& C_{d_{i} d_{j}}^{V, A}=\frac{1}{2 N}\left(V_{d_{L}}^{\dagger} \chi_{Q_{L}} V_{d_{L}} \pm V_{d_{R}}^{\dagger} X_{d_{R}} V_{d_{R}}\right)_{i j} .
\end{aligned}
$$

\footnotetext{
${ }^{1}$ The diagonal vector couplings do not contribute to on-shell physical processes, as it can be seen upon integrating by parts and using the equations of motion.
}

Note that the unitary flavour matrices are only subject to the constraint $V_{\mathrm{CKM}}=V_{u_{L}}^{\dagger} V_{d_{L}}$.

A common assumption, as e.g. in the original Peccei-Quinn-Weinberg-Wilczek (PQWW) [10-13] and the Dine-Fischler-Srednicki-Zhitnitsky (DFSZ) [14, 15] axion models, consists in the universality of the PQ current, i.e. $\mathcal{X}_{Q_{L}, u_{R}, d_{R}} \propto \mathbb{\Omega}_{3} .{ }^{2}$ This implies flavour blind axion interactions: the axion interacts in the same way with SM fermions having the same gauge quantum numbers and, moreover, off-diagonal entries in Eq. (5) trivially vanish.

\section{A lesson from flavour}

The simplest axion model featuring only two Higgs doublets for the breaking of the $\mathrm{U}(1)_{\mathrm{PQ}}$, also known as PQWW model, was ruled out quite soon after its conception, due to a combination of beam dump experiments and rare meson decays such as $K \rightarrow \pi a$ [18] and Quarkonia $\rightarrow \gamma a$ [19]. For instance, radiative decays of Quarkonia to $\gamma a$ normalized to leptonic modes can be written at the leading order as [19]

$$
\frac{\mathcal{B}(J / \psi \rightarrow \gamma a)}{\mathcal{B}(J / \psi \rightarrow \mu \mu)}=\frac{g_{c}^{2}}{2 \pi \alpha}, \quad \frac{\mathcal{B}(\Upsilon \rightarrow \gamma a)}{\mathcal{B}(\Upsilon \rightarrow \mu \mu)}=\frac{g_{b}^{2}}{2 \pi \alpha},
$$

where $g_{c}=m_{c} C_{c c}^{A} / f_{a}$ (in the notation of Eq. (5)) and similarly for $g_{b}$. In the PQWW model one has

$$
g_{c}=\frac{m_{c}}{v} \frac{1}{\tan \beta}, \quad g_{b}=\frac{m_{b}}{v} \tan \beta,
$$

with $v=246 \mathrm{GeV}$ and $\tan \beta=\left\langle H_{u}\right\rangle /\left\langle H_{d}\right\rangle$. Since the couplings in Eq. (9) cannot be simultaneously suppressed, bounds from Quarkonia alone would have been sufficient to rule out the PQWW model (for a historical account see Sect. 3 in Ref. [20]).

However, a main assumption behind the PQWW model consisted in the universality of the PQ current. At the beginning of the 80 's it seemed more natural to keep this assumption, and extend the model by adding a SM-singlet field in order to decouple the PQ breaking from the electroweak scale. ${ }^{3}$ Models of these type became known as invisible axion models and led to the standard KSVZ/DFSZ benchmarks. In the same years, the GSI anomaly [21-23] (a sharp peak in the $e^{+}$spectrum of heavy ion collisions) which could be interpreted in terms of an $O(\mathrm{MeV})$ axion with dominant decay mode $a \rightarrow e^{+} e^{-}$, triggered a lot of interest in trying to explain that in terms of variant axion models, with a non-universal axion mainly coupled to first generation fermions in order to avoid bounds from Quarkonia decays. It actually took almost a decade, starting from the original PQWW proposal, to rule out non-universal PQWW variants, by combining informations from rare $\pi$ and $K$ decays [24]. Even nowadays, there is an interesting claim that under certain

\footnotetext{
${ }^{2}$ We remind the reader that instead in Kim-Shifman-VainshteinZakharov (KSVZ) $[16,17]$ type of models the SM fields are not charged under the $\mathrm{U}(1)_{\mathrm{PQ}}$.

${ }^{3}$ On the contrary, it can be argued that imposing $f_{a} \gg v$ leads to two well-known naturalness issues: the hierarchy problem and the PQ-quality issue.
} 
conditions (among which the non-universality of the PQ current) an $O(\mathrm{MeV})$ axion is not obviously ruled out [25]. ${ }^{4}$

\section{Non-universal Peccei-Quinn axion models}

We explore now some consequences of non-universal PQ axion models, focussing on some recent developments such as the possibility of suppressing the axion coupling to protons and neutrons (nucleophobia), which allows in turn to relax the Supernova (SN) bound on the axion mass. We next make some considerations on possible connections of non-universal PQ scenarios with the SM flavour puzzle and summarize the status of flavoured axion searches.

\subsection{Nucleophobia}

The axion coupling to nucleons (cf. Eq. (2)) can be computed using a non-relativistic effective field theory where nucleons are at rest and the axion is treated as an external current (see [5] for details). In particular, one obtains

$$
\begin{aligned}
& C_{p}+C_{n}=\left(c_{u}+c_{d}\right)\left(\Delta_{u}+\Delta_{d}\right)-2 \delta_{s}, \\
& C_{p}-C_{n}=\left(c_{u}-c_{d}\right)\left(\Delta_{u}-\Delta_{d}\right),
\end{aligned}
$$

where $c_{u, d}$ are derivative axion couplings to the axial current of valence quarks in the nucleon, defined in the basis where the $a G \tilde{G}$ has been rotated away (cf. Eq. (13)), $\delta_{s} \approx 5 \%$ encodes effects from sea quarks in the nucleon, and $\Delta_{u, d}$ are nucleon matrix elements which are extracted from experiments and/or the Lattice. Hence, if we want to simultaneously suppress both the axion-nucleon couplings, say at the level of $10 \%$, we need to impose $c_{u}+c_{d}=0$ and $c_{u}-c_{d}=0$, regardless of the specific value of $\Delta_{u, d}$.

It is instructive to see why achieving such cancellation is not possible in standard KSVZ and DFSZ models. Let us consider the axion Lagrangian in Eq. (4), restricted to first generation up and down quarks, and ignoring for the moment flavour mixing. Including also the $a G \tilde{G}$ term, the latter reads

$$
\mathcal{L}_{a} \supset \frac{a}{f_{a}} \frac{\alpha_{a}}{8 \pi} G \tilde{G}+\frac{\partial_{\mu} a}{v_{\mathrm{PQ}}}\left[\mathcal{X}_{u} \bar{u} \gamma^{\mu} \gamma_{5} u+\mathcal{X}_{d} \bar{d} \gamma^{\mu} \gamma_{5} d\right]
$$

where $2 X_{u} \equiv X_{Q_{1}}-X_{u_{1}}$ and $2 X_{d} \equiv X_{Q_{1}}-X_{d_{1}}$ are the $\mathrm{PQ}$ charges expressed in terms of the chiral ones (for notational simplicity, we have suppressed the chirality indices in the right-hand sides). The $a G \tilde{G}$ term can be rotated away via a field-dependent 2 -flavour quark transformation: $q \rightarrow \exp \left(i \gamma_{5} Q_{a} a(x) /\left(2 f_{a}\right)\right) q$, with $q=(u, d)^{T}$ and $Q_{a}=\operatorname{diag}\left(m_{d} /\left(m_{u}+m_{d}\right), m_{u} /\left(m_{u}+m_{d}\right)\right)$. The latter choice ensures that the axion has no mass mixing with the neutral

\footnotetext{
${ }^{4}$ This is not in contradiction with the previous statement, since the UV completion of this axion is not of the PQWW type.
}

pion [26]. In the new basis, Eq. (12) reads

$$
\begin{gathered}
\mathcal{L}_{a} \supset \frac{\partial_{\mu} a}{2 f_{a}}[\underbrace{\left(\frac{X_{u}}{N}-\frac{m_{d}}{m_{u}+m_{d}}\right)}_{c_{u}} \bar{u} \gamma^{\mu} \gamma_{5} u \\
+\underbrace{\left(\frac{X_{d}}{N}-\frac{m_{u}}{m_{u}+m_{d}}\right)}_{c_{u}} \bar{d} \gamma^{\mu} \gamma_{5} d] .
\end{gathered}
$$

Hence, the nucleophobic conditions can be recast as

$$
\begin{aligned}
& 0=c_{u}+c_{d}=\frac{\mathcal{X}_{u}+\mathcal{X}_{d}}{N}-1, \\
& 0=c_{p}-c_{n}=\frac{\mathcal{X}_{u}-\mathcal{X}_{d}}{N}-\frac{m_{d}-m_{u}}{m_{u}+m_{d}} .
\end{aligned}
$$

While the second condition can always be implemented via a tuning (see below), the real bottleneck is the first one, since $\mathcal{X}_{u}=\mathcal{X}_{d}=0$ in KSVZ models, while in DFSZ models one has $N=\frac{1}{2} n_{f}\left(2 X_{Q_{1}}-\mathcal{X}_{u_{1}}-\mathcal{X}_{d_{1}}\right)=n_{f}\left(\mathcal{X}_{u}+\mathcal{X}_{d}\right)$, with $n_{f}=3$ denoting the number of families.

The above no-go theorem for standard axion models suggests itself a possible way out [27]: if the total anomaly factor were equal to that of the first family, i.e. $N=N_{1}=$ $X_{u}+\mathcal{X}_{d}$, the first condition would be automatically satisfied. To simplify the discussion, let us assume a $2+1$ structure such that the PQ charges of the first two generations the same. Then the condition we would like to impose, $N \equiv N_{1}+N_{2}+N_{3}=N_{1}$, simply implies $N_{1}=N_{2}=-N_{3}$. It is remarkably simple to obtain the latter in terms of a renormalizable Yukawa Lagrangian featuring two Higgs doublets $H_{1,2} \sim(1,2,-1 / 2)$. For instance,

$$
\begin{aligned}
\mathcal{L}_{Y} & =\bar{Q}_{3} u_{3} H_{1}+\bar{Q}_{3} d_{3} \tilde{H}_{2}+\ldots+\bar{Q}_{2} u_{2} H_{2}+\bar{Q}_{2} d_{2} \tilde{H}_{1}+\ldots \\
& +\bar{Q}_{1} u_{1} H_{2}+\bar{Q}_{1} d_{1} \tilde{H}_{1}+\ldots,
\end{aligned}
$$

where we have suppressed Yukawa couplings and the dots stand for off-diagonal operators which are necessary in order to obtain a realistic CKM mixing, compatibly the overall PQ charge assignments. ${ }^{5}$ Denoting by $X_{1,2}$ the PQ charges of $H_{1,2}$, from Eq. (16) we have $N_{3}=\frac{1}{2}\left(2 X_{Q_{3}}-X_{u_{3}}-X_{d_{3}}\right)=\frac{1}{2}\left(X_{1}-X_{2}\right)$ and $N_{2}=$ $\frac{1}{2}\left(2 X_{Q_{2}}-X_{u_{2}}-X_{d_{2}}\right)=\frac{1}{2}\left(X_{2}-X_{1}\right)=-N_{3}$, having swapped the role of $H_{1}$ and $H_{2}$ for the third and second/first generations. Hence, $N=N_{1}=\frac{1}{2}\left(2 \mathcal{X}_{Q_{1}}-\mathcal{X}_{u_{1}}-\mathcal{X}_{d_{1}}\right)=\mathcal{X}_{u}+\mathcal{X}_{d}$ and Eq. (14) is automatically satisfied in terms of charge assignments.

Coming to the second nucleophobic condition in Eq. (15), this can be expressed as a condition on $\tan \beta=$ $\left\langle H_{2}\right\rangle /\left\langle H_{1}\right\rangle$. In order to see that, let us first impose the orthogonality between the PQ and hypercharge currents: $Y\left(H_{1}\right) X_{1} v_{1}^{2}+Y\left(H_{2}\right) X_{2} v_{2}^{2}=0$, and hence $\mathcal{X}_{1} / \mathcal{X}_{2}=-\tan ^{2} \beta$, implying no kinetic mixing between the axion and the $Z$ boson. Using $X_{u}-X_{d}=\frac{1}{2}\left(X_{d_{1}}-X_{u_{1}}\right)=\frac{1}{2}\left(X_{2}+X_{1}\right)$ and

\footnotetext{
${ }^{5}$ This include as well a scalar potential communicating the PQ breaking to the two Higgs doublets via a SM singlet scalar, in a fashion similar to universal DFSZ models. In the absence of texture zeros and for a $2+1$ structure for the PQ charges, all the possible Yukawa structures leading to nucleophobia have been classified in Ref. [27]. The opposite case of maximal numbers of texture zeros has been discussed instead in Ref. [28].
} 
$N=N_{1}=\frac{1}{2}\left(2 X_{Q_{1}}-\mathcal{X}_{u_{1}}-\mathcal{X}_{d_{1}}\right)=\frac{1}{2}\left(\mathcal{X}_{2}-\mathcal{X}_{1}\right)$, we can recast Eq. (15) as $\left(X_{2}+X_{1}\right) /\left(X_{2}-\mathcal{X}_{1}\right)=\cos ^{2} \beta-\sin ^{2} \beta=\left(m_{d}-\right.$ $\left.m_{u}\right) /\left(m_{u}+m_{d}\right) \approx 1 / 3$, which is satisfied for $\tan \beta \approx 1 / \sqrt{2}$.

To sum up, with a single $O(10 \%)$ tuning, ${ }^{6} \tan \beta \approx$ $1 / \sqrt{2}$, one can simultaneously suppress both the axion coupling to neutrons and protons, which allows in turn to relax the SN bound by one order of magnitude compared to standard KSVZ/DFSZ scenarios, e.g. in KSVZ models the often quoted bound reads $m_{a} \lesssim 0.02 \mathrm{eV}$ [29]. However, recent analyses of the axion emissivity from the SN core hint to a weakening of the bound by a factor of a few [30, 31].

It should be noted that in the construction above the axion-electron coupling, $g_{a e}$, turns out to be generically sizeable, ${ }^{7}$ and additional strong bounds on $m_{a}$ are obtained from white-dwarf cooling rates and red giants evolution in globular clusters. Hence, in order to effectively relax astrophysical bounds on $m_{a}$, also $g_{a e}$ must be somewhat suppressed. This can be done at the cost of an extra tuning with flavour mixing in the leptonic sector [27], or, more elegantly, by introducing a leptonic Higgs doublet $\mathrm{H}_{3}$ which allows for a correlated cancellation of the axion coupling to nucleons and electrons via a single tuning [33]. This kind of scenarios have been dubbed astrophobic, in the sense that all the main astrophysical constraints can be relaxed up to one order of magnitude, allowing for axions as heavy as $m_{a} \sim 0.2 \mathrm{eV}$. On the other hand, the axion coupling to photons remains generically sizeable, e.g. $E / N=\{-4 / 3,2 / 3,8 / 3,14 / 3\}$ in the models of Ref. [27], so that the next generation of helioscopes, such as IAXO [34], will be able to probe their parameter space.

\subsection{Connections with the Standard Model flavour puzzle}

We have seen in the previous section that nucleophobia provides a clear case for the non-universality of the PQ current, but it does not fix the size of flavour mixing entering into the axion couplings to SM fermions when going to the mass basis (cf. Eqs. (6)-(7)). The diagonalizing flavour matrices, subject only to the constraint $V_{\mathrm{CKM}}=V_{u_{L}}^{\dagger} V_{d_{L}}$, are in principle free parameters which can be measured in flavour violating (FV) processes involving the axion. Extra theoretical bias, possibly in connection with the SM flavour puzzle, can help in providing an organizing principle for the flavour structure. A particularly natural realization is to identify the $\mathrm{U}(1)_{\mathrm{PQ}}$ with the horizontal $\mathrm{U}(1)$ symmetry responsible for the Yukawa hierarchies [35-38]. ${ }^{8}$ Models of Froggatt-Nielsen [43] type have recently regained some attention (see e.g. [44, 45]), and they typically predict axion flavour transitions controlled by the

\footnotetext{
${ }^{6}$ The level of tuning useful for nucleophobia is limited by the remainder $2 \delta_{s} \approx 10 \%$ in Eq. (10), which sets an irreducible contribution to nucleon couplings. In principle, flavour mixing (neglected in the present simplified discussion) also enters diagonal axion couplings when going to the mass basis (cf. Eqs. (6)-(7)), and it can be used to further cancel the $\delta_{s}$ contribution [27].

${ }^{7}$ Having a sizeable $g_{a e}$ with a somewhat relaxed $\mathrm{SN}$ bound improves the fit of the cooling anomalies [32].

${ }^{8}$ Gauging the horizontal flavour group can also lead to an accidental global U(1) whose spontaneous breaking delivers a flavoured arion [39] or a flavoured axion [40-42].
}

CKM matrix, although subject to irreducible $O(1)$ uncertainties common also to other flavour models. A different kind of approach $[28,46]$, based on requiring the maximal number of textures zeros (compatibly with SM fermion masses and mixings) that can be enforced via a family dependent $\mathrm{U}(1)_{\mathrm{PQ}}$ at the renormalizable level, allows instead to completely fix ${ }^{9}$ the axion couplings to SM fermions, including off-diagonal ones, in terms of a fit to SM fermion masses and mixings.

\subsection{Flavoured axion searches}

Rare FV decays with invisible and light final states allow to probe the off-diagonal axion couplings $C_{\psi_{i} \psi_{j}}^{A, V}$ in Eq. (5). A collection of bounds can be found for instance in Refs. [47-49]. The strongest limits on FV axion couplings to quarks come from $K^{+} \rightarrow \pi^{+} a$. Comparing the theoretical prediction with the current limit from E949/E787 [50] gives $m_{a}<2 \cdot 10^{-5} \mathrm{eV} /\left|C_{s d}^{V}\right|$, which for maximal mixing (i.e. $C_{s d}^{V}=O(1)$ ) is about three orders of magnitude stronger than typical astrophysical bounds. Hence, $K^{+} \rightarrow \pi^{+} a$ clearly provides a golden channel for FV axion searches. ${ }^{10}$ NA62 is expected to improve the limit on $\mathcal{B}\left(K^{+} \rightarrow \pi^{+} a\right)$ by a factor of $\sim 70$ [51, 52], thus strengthening the axion mass bound by a factor $\sim 8$. The next most sensitive process in the quark sector is $B^{+} \rightarrow K^{+} a$, whose present limit from CLEO [53] corresponds to $m_{a}<9 \cdot 10^{-2} \mathrm{eV} /\left|C_{b s}^{V}\right|$, close to astrophysical limits for maximal mixing. This latter bound could be presumably strengthened by a factor $\sim 10$ at BELLE II [54]. A similar, but slightly weaker bound, is obtained for $B^{+} \rightarrow \pi^{+} a$, which involves the coupling $C_{b d}^{V}$. On the other hand, bounds on processes involving other quark transitions are about three orders of magnitude smaller (see Table 2 in Ref. [48]), and hence not competitive with astrophysical limits, even for maximal mixing. In the charged lepton sector, the strongest limits come from (30 years old) searches for $\mu \rightarrow$ eya $[55,56]$. They yield $m_{a}<3 \cdot 10^{-3} \mathrm{eV} /\left(\left|\left(C_{\mu e}^{V}\right)^{2}+\right|\left(C_{\mu e}^{A}\right)^{2} \mid\right)^{1 / 2}$, competitive with astrophysical bounds in the case of sizeable flavour mixing. This bound is likely to be improved by one order of magnitude at the MEG [57] and Mu3e [58] experiments at PSI. On the other hand, bounds on $\tau$ - $\mu$ and $\tau$-e transitions from ARGUS [59] yield axion mass limits which are still well-below astrophysical ones.

\section{Conclusions}

Relaxing the assumption of the universality of the PQ current opens up the parameters space of DFSZ models, by

\footnotetext{
${ }^{9} \mathrm{Up}$ to the usual dependence from $f_{a}$ and $\tan \beta$, as in universal DFSZ models.

${ }^{10}$ It should be noted that pseudo-scalar meson decays such as $K \rightarrow$ $\pi a$ are sensitive only to the vectorial part of the quark current, since $\left\langle\pi\left|\bar{s} \gamma_{\mu} \gamma_{5} d\right| K\right\rangle=0$ by the Wigner-Eckart theorem. In order to set bounds on $C_{s d}^{A}$ one has to resort to other FV processes, as for example $K^{0}-\bar{K}^{0}$ mixing, which however are much weaker compared to the limits on the vectorial counterpart from meson decays. In principle, this would leave open a possibility in order to evade the strong constraints from $K \rightarrow \pi a$ in the presence of large mixing, if one could cook up a model with $C_{s d}^{V} \ll C_{s d}^{A}$
} 
introducing 34 new real parameters only in the quark sector (most of which are related to the diagonalizing $\mathrm{U}(3)$ matrices in Eqs. (6)-(7)). These new parameters arise, in some sense, within the SM (since they come from the diagonalization of the Yukawas that we write in the SM Lagrangian), but the SM is "blind" to them. By extending the $\mathrm{SM}$ with the axion at low-energy, it is somewhat artificial to require that flavour mixing beyond the CKM is unphysical and we should rather let experiments to decide. In the meanwhile, it is worth to speculate about the possible consequences of the non-universality of the PQ current, as I have done in the present contribution.

To conclude, I cannot resist from making an unfortunate analogy: "DFSZ = cMSSM". The DFSZ model corresponds, in some sense, to the constrained version of the MSSM with universal soft terms. There is however an important difference, while large flavour violating effects in low-energy SUSY were often considered as a curse (flavour problem), in the case of the axion they rather come as a blessing (flavour opportunity).

\section{Acknowledgments}

I thank the organizers of the FCCP19 workshop, and in particular Giancarlo D'Ambrosio, for the warm hospitality. I also thank Fred Björkeroth, Federico Mescia, Enrico Nardi, Paolo Panci and Robert Ziegler for an enjoyable collaboration on flavoured axions. This work is supported by the Marie Skłodowska-Curie Individual Fellowship grant AXIONRUSH (GA 840791).

\section{References}

[1] L. Di Luzio, M. Giannotti, E. Nardi, L. Visinelli (2020), 2003.01100

[2] I.G. Irastorza, J. Redondo, Prog. Part. Nucl. Phys. 102, 89 (2018), 1801.08127

[3] C. Vafa, E. Witten, Phys. Rev. Lett. 53, 535 (1984)

[4] M. Gorghetto, G. Villadoro, JHEP 03, 033 (2019), 1812.01008

[5] G. Grilli di Cortona, E. Hardy, J. Pardo Vega, G. Villadoro, JHEP 01, 034 (2016), 1511.02867

[6] M. Srednicki, Nucl. Phys. B260, 689 (1985)

[7] S. Chang, K. Choi, Phys. Lett. B316, 51 (1993), hep-ph/9306216

[8] L. Di Luzio, F. Mescia, E. Nardi, Phys. Rev. Lett. 118, 031801 (2017), 1610.07593

[9] L. Di Luzio, F. Mescia, E. Nardi, Phys. Rev. D96, 075003 (2017), 1705.05370

[10] R.D. Peccei, H.R. Quinn, Phys. Rev. Lett. 38, 1440 (1977)

[11] R.D. Peccei, H.R. Quinn, Phys. Rev. D16, 1791 (1977)

[12] S. Weinberg, Phys. Rev. Lett. 40, 223 (1978)

[13] F. Wilczek, Phys. Rev. Lett. 40, 279 (1978)

[14] A.R. Zhitnitsky, Sov. J. Nucl. Phys. 31, 260 (1980), [Yad. Fiz.31,497(1980)]

[15] M. Dine, W. Fischler, M. Srednicki, Phys. Lett. B104, 199 (1981)
[16] J.E. Kim, Phys. Rev. Lett. 43, 103 (1979)

[17] M.A. Shifman, A.I. Vainshtein, V.I. Zakharov, Nucl. Phys. B166, 493 (1980)

[18] L.J. Hall, M.B. Wise, Nucl. Phys. B187, 397 (1981)

[19] F. Wilczek, Phys. Rev. Lett. 39, 1304 (1977)

[20] M. Davier, Searches for New Particles, in Proceedings, 23RD International Conference on High Energy Physics, JULY 16-23, 1986, Berkeley, CA (1986)

[21] J. Schweppe et al., Phys. Rev. Lett. 51, 2261 (1983)

[22] M. Clemente, E. Berdermann, P. Kienle, H. Tsertos, W. Wagner, C. Kozhuharov, F. Bosch, W. Konig, Phys. Lett. 137B, 41 (1984)

[23] T. Cowan et al., Phys. Rev. Lett. 54, 1761 (1985)

[24] W.A. Bardeen, R.D. Peccei, T. Yanagida, Nucl. Phys. B279, 401 (1987)

[25] D.S.M. Alves, N. Weiner, JHEP 07, 092 (2018), 1710.03764

[26] H. Georgi, D.B. Kaplan, L. Randall, Phys. Lett. 169B, 73 (1986)

[27] L. Di Luzio, F. Mescia, E. Nardi, P. Panci, R. Ziegler, Phys. Rev. Lett. 120, 261803 (2018), 1712 . 04940

[28] F. Bjorkeroth, L. Di Luzio, F. Mescia, E. Nardi, JHEP 02, 133 (2019), 1811 . 09637

[29] M. Tanabashi et al. (Particle Data Group), Phys. Rev. D98, 030001 (2018)

[30] J.H. Chang, R. Essig, S.D. McDermott, JHEP 09, 051 (2018), 1803.00993

[31] P. Carenza, T. Fischer, M. Giannotti, G. Guo, G. Martinez-Pinedo, A. Mirizzi, JCAP 1910, 016 (2019), 1906. 11844

[32] M. Giannotti, I.G. Irastorza, J. Redondo, A. Ringwald, K. Saikawa, JCAP 1710, 010 (2017), 1708.02111

[33] F. Bjorkeroth, L. Di Luzio, F. Mescia, E. Nardi, P. Panci, R. Ziegler (2019), 1907.06575

[34] E. Armengaud et al. (IAXO), JCAP 1906, 047 (2019), 1904.09155

[35] A. Davidson, K.C. Wali, Phys. Rev. Lett. 48, 11 (1982)

[36] F. Wilczek, Phys. Rev. Lett. 49, 1549 (1982)

[37] A. Davidson, V.P. Nair, K.C. Wali, Phys. Rev. D29, 1504 (1984)

[38] A. Davidson, M.A.H. Vozmediano, Nucl. Phys. B248, 647 (1984)

[39] Z.G. Berezhiani, Phys. Lett. 129B, 99 (1983)

[40] Z.G. Berezhiani, Phys. Lett. 150B, 177 (1985)

[41] Z.G. Berezhiani, M.Yu. Khlopov, R.R. Khomeriki, Sov. J. Nucl. Phys. 52, 344 (1990), [Yad. Fiz.52,538(1990)]

[42] Z.G. Berezhiani, M.Yu. Khlopov, Z. Phys. C49, 73 (1991)

[43] C.D. Froggatt, H.B. Nielsen, Nucl. Phys. B147, 277 (1979)

[44] Y. Ema, K. Hamaguchi, T. Moroi, K. Nakayama, JHEP 01, 096 (2017), 1612 . 05492 
[45] L. Calibbi, F. Goertz, D. Redigolo, R. Ziegler, J. Zupan, Phys. Rev. D95, 095009 (2017), 1612.08040

[46] F. Bjorkeroth, L. Di Luzio, F. Mescia, E. Nardi (2019), 1910.00576

[47] J.L. Feng, T. Moroi, H. Murayama, E. Schnapka, Phys. Rev. D57, 5875 (1998), hep-ph/9709411

[48] F. Bjorkeroth, E.J. Chun, S.F. King, JHEP 08, 117 (2018), 1806.00660

[49] R. Ziegler, PoS CORFU2018, 035 (2019), 1905.01084

[50] S. Adler et al. (E949, E787), Phys. Rev. D77, 052003 (2008), 0709. 1000

[51] G. Anelli et al. (2005)
[52] R. Fantechi (NA62), The NA62 experiment at CERN: status and perspectives, in 12th Conference on Flavor Physics and CP Violation (FPCP 2014) Marseille, France, May 26-30, 2014 (2014), 1407.8213

[53] R. Ammar et al. (CLEO), Phys. Rev. Lett. 87, 271801 (2001), hep-ex/0106038

[54] T. Abe et al. (Belle-II) (2010), 1011.0352

[55] R.D. Bolton et al., Phys. Rev. D38, 2077 (1988)

[56] J.T. Goldman et al., Phys. Rev. D36, 1543 (1987)

[57] F. Renga (MEG), Hyperfine Interact. 239, 58 (2018), 1811.05921

[58] A. Blondel et al. (2013), 1301.6113

[59] H. Albrecht et al. (ARGUS), Z. Phys. C68, 25 (1995) 